\title{
Imaging charge transfer in crystals using electron ptychography
}

Peter Nellist $^{1}$, Gerardo Martinez ${ }^{1}$, Timothy Pennycook ${ }^{2}$, Lewys Jones ${ }^{1}$, Hao Yang ${ }^{3}$, Martin Huth ${ }^{4}$, Martin Simson ${ }^{4}$, Heike Soltau ${ }^{4}$, Yukihito Kondo ${ }^{5}$, Ryusuke Sagawa ${ }^{5}$

${ }^{1}$ Department Of Materials, University Of Oxford, Oxford, United Kingdom, ${ }^{2}$ Faculty of Physics, University of Vienna, Vienna, Austria,

${ }^{3}$ Molecular Foundry, Lawrence Berkeley National Laboratory, Berkeley, United States, ${ }^{4}$ PNDetector GmbH, Muenchen, Germany,

5JEOL Ltd., Tokyo, Japan

E-mail: peter.nellist@materials.ox.ac.uk

Electron ptychography is a phase retrieval method that allows for the formation of phase images in the scanning transmission electron microscope (STEM). A potential application of such phase imaging is the direct detection of charge transfer due to bonding in crystals.

Recent developments in fast pixelated detectors have enabled the acquisition of 4D-datasets that contain the full convergent beam electron diffraction pattern for each probe position in a STEM scan. Using a ptychographical reconstruction, such as the Wigner Distribution Deconvolution (WDD), it has been shown that quantitative phase images can be formed, along with the correction of residual aberrations [1]. Phase contrast images have been shown to be sensitive to the electronic charge distribution and the effects of bonding [2], but these measurements can be affected by small residual lens aberrations, which affect the image contrast and can overwhelm the small signal due to charge transfer. Here we use the ability to correct residual lens aberrations post-acquisition to explore the visualisation of charge transfer in monolayer hexagonal boron nitride (hBN).

We acquired a ptychographic dataset of monolayer hBN using an aberration-corrected JEOL ARM200CF instrument fitted with a pnCCD (S)TEM camera. The acceleration voltage was $60 \mathrm{kV}$ to avoid damage and the 4D datasets were acquired at 1000 frames per second. Aberration free multislice image simulations considering the so-called independent atom model (IAM) with the experimental dose settings were calculated using the MULTEM software [3]. Figure 1 a) shows the aberration corrected experimental phase difference obtained from a WDD ptychographic reconstruction. Figure 1 b) shows the reconstruction of the simulation assuming a source size broadening effect of FWHM 1.05 Angstrom. The source size broadening was estimated to match the maximum experimental phase difference. 2D Gaussian functions were fitted to each $\mathrm{N}$ and $\mathrm{B}$ atom positions in the reconstructed phase images for both experiment and simulations. The heights of the fitted 2D Gaussian functions were used as measure of the phase difference caused by the atoms. They are plotted as histograms in Figure $1 \mathrm{c}$ ), and d), to which a Gaussian function was fitted to determine the mean difference between the phase difference distributions. From histogram $1 \mathrm{c}$ ), the mean difference between phase differences at $\mathrm{N}$ and $\mathrm{B}$ sites is only 2.3 mrad. For the case of simulations, a mean difference of $5.5 \mathrm{mrad}$ phase difference.

When phase imaging $\mathrm{hBN}$, it is expected that the contrast of $\mathrm{N}$ sites will be reduced due to the screening of the nuclear potential on the $\mathrm{N}$ species caused by the accumulation of charge transferred from the $\mathrm{B}$ site. The result from ptychography suggests that this charge transfer is being directly detected, and creates the opportunity to examine charge transfer at features such as vacancies and edges in the monolayer sheet.

[1] Yang H. et al. (2016), Nature Communications, 7, 12532.

[2] Meyer J.C. et al. (2011) Nature Materials, 10, 209.

[3] Lobato, I. \& Van Dyck, D. (2015). Ultramicroscopy, 156, 9.

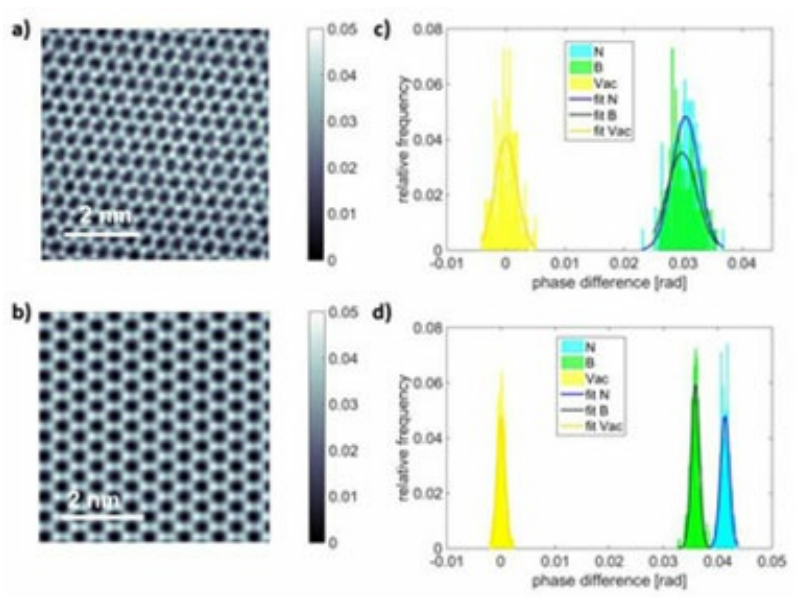

Keywords: ptychography, phase imaging, STEM 